\title{
BMJ Open Effects of vestibular rehabilitation, with or without betahistine, on managing residual dizziness after successful repositioning manoeuvres in patients with benign paroxysmal positional vertigo: a protocol for a randomised controlled trial
}

\author{
Peixia Wu, ${ }^{\oplus 1}$ Wenzhu Cao, ${ }^{2}$ Yan $\mathrm{Hu},{ }^{3}$ Huawei $\mathrm{Li}^{4}$
}

To cite: Wu P, Cao W, Hu Y, et al. Effects of vestibular rehabilitation, with or without betahistine, on managing residual dizziness after successful repositioning manoeuvres in patients with benign paroxysmal positional vertigo: a protocol for a randomised controlled trial. BMJ Open 2019;9:e026711. doi:10.1136/ bmjopen-2018-026711

- Prepublication history for this paper is available online. To view these files, please visit the journal online (http://dx.doi. org/10.1136/bmjopen-2018026711).

$\mathrm{YH}$ and $\mathrm{HL}$ contributed equally.

Received 20 September 2018

Revised 2 March 2019

Accepted 29 April 2019

Check for updates

(C) Author(s) (or their employer(s)) 2019. Re-use permitted under CC BY-NC. No commercial re-use. See rights and permissions. Published by BMJ.

For numbered affiliations see end of article.

Correspondence to Professor Yan Hu; fudanhuyan@163.com and Professor Huawei Li; hwli@shmu.edu.cn

\begin{abstract}
Introduction Benign paroxysmal positional vertigo (BPPV) is recognised as the leading cause of peripheral vertigo in adults. The canalith repositioning procedure (CRP) can be used for effective treatment of BPPV. However, some patients experience residual dizziness (RD) even after successful CRP, resulting in a significant negative impact on their daily function and quality of life. Exercise-based vestibular rehabilitation (VR) has been proven as an effective method for managing dizziness and has been applied in patients with various vestibular disorders. However, the efficacy of VR to specifically target RD post-BPPV is unknown. This study aims to investigate the efficacy of VR, compared with betahistine or VR plus betahistine treatment, in the treatment of patients experiencing RD after successful CRP.
\end{abstract}

Methods and analysis A randomised single-blinded controlled trial will be carried out to determine the efficacy of VR compared with betahistine or VR plus betahistine treatment in mitigating $\mathrm{RD}$ and improving balance function. Patients with BPPV who experience RD after successful CRP will be recruited. Participants will be randomised into one of three groups to receive VR, betahistine or VR plus betahistine. There will be 61 participants in each group. The primary outcomes will be changes in the patient's daily function as measured by the Vestibular Activities and Participation questionnaire and balance ability assessed by computerised dynamic posturography. The secondary outcomes will be dizziness-related handicap, otolith function and duration of RD symptoms. Outcome measures will be noted at baseline and at 2, 4 and 8 weeks postrandomisation. This study has the potential to reduce unnecessary anti-vertigo drug prescriptions and may lead to a general consensus regarding the use of VR as a firstline treatment for RD in patients with BPPV.

Ethics and dissemination This trial received ethical approval from the Institutional Review Board of Eye and ENT Hospital of Fudan University (reference number 2017046). The study results will be disseminated via peerreviewed journals and conferences.

\section{Strengths and limitations of this study}

To the best of our knowledge, this is the first randomised controlled trial to evaluate the effects of vestibular rehabilitation (VR), compared with betahistine or VR in addition to betahistine, on managing residual dizziness after successful canalith repositioning procedure in patients with benign paroxysmal positional vertigo.

- Both subjective and objective outcome measures will be comprehensively assessed with a reasonably long follow-up period. In particular, patients' activities and participation aspect based on the International Classification of Functioning, Disability and Health framework will be evaluated. Such a trial will be able to fully reflect the efficacy of the proposed intervention.

- For practical reasons, there will be no control group with sham, placebo or no treatment in this study; therefore, the study will not be useful to determine whether participants improve spontaneously or to determine the mechanisms by which the three interventions may function to alter outcomes.

Trial registration number NCT03624283; Pre-results.

\section{INTRODUCTION}

Benign paroxysmal positional vertigo (BPPV) is the leading cause of peripheral vertigo, with a prevalence of 10.7-64 per 100000 people and a lifetime prevalence of $2.4 \% .{ }^{1}$ The time at onset is most commonly between the fifth and seventh decade of life, but BPPV can occur at any age. ${ }^{2}$ Two hypotheses, involving cupulolithiasis $^{3}$ and canalithiasis, ${ }^{4}$ are among the most widely accepted hypotheses that elucidate the pathophysiology of BPPV. The 
hypothesis involving canalithiasis has gained wider acceptance in the scientific field because it offers a better explanation of the symptoms. ${ }^{5}$ According to the canalithiasis hypothesis, BPPV is caused by free otoconia dislodging from the utricular macula and entering the semicircular canal, which promotes inappropriate flow of endolymph whenever the head is rotated in the plane of the affected canal. ${ }^{4}$ Although any of the three semicircular canals can be affected, the posterior semicircular canal is the most common, followed by the horizontal canal. Treatment for BPPV consists of the canalith repositioning procedure (CRP) with the aim of repositioning the displaced particles from the affected canal to their original location. ${ }^{6}$ The CRP has long been a mainstay therapy and is now well established as the gold treatment for BPPV. ${ }^{7}$ Posterior canal BPPV can be treated by performing Epley's manoeuvre or Semont's liberatory manoeuvre. For horizontal canal BPPV, barbecue rotation or the Gufoni manoeuvre have been demonstrated to be effective.

Despite the fact that the CRP can provide rapid relief of vertigo in patients with BPPV, residual symptoms may remain even after the disappearance of typical vertigo and nystagmus following a successful CRP. These residual symptoms, also referred to as residual dizziness (RD), imply a non-specific sensation of unsteadiness, lightheadedness, disorientation, fogginess, or drowsiness. ${ }^{8}$ The reported incidence of RD ranges from $29.6 \%$ to $76.9 \%$, and the duration of RD can range from a few days to several months. ${ }^{8-12}$ Several authors have reported that RD symptoms may persist for $>3$ months, ${ }^{13} 14$ resulting in adverse physical and psychological consequences.

Different pathophysiological mechanisms have been proposed to explain the presence of $\mathrm{RD}$, but the cause remains unknown. Previous studies have suggested the following possible explanations: (1) the persistence of debris in the canal is insufficient to provoke detectable positional nystagmus or vertigo ${ }^{11}$; (2) otolith dysfunction $^{15}{ }^{16}$; (3) coexisting vestibular disease that leads to incomplete central adaptation; (4) potential autonomic dysfunction such as orthostatic hypotension ${ }^{17}$ and (5) persistent postural-perceptual dizziness. ${ }^{18}$

Dizziness increases the risk of falls or fear of falling, disturbs daily life and results in restriction of social activities. As a result, many patients with BPPV tend to avoid certain activities, limit their movement and avoid specific circumstances due to fear of provoking dizziness and unanticipated unsteadiness attacks. ${ }^{19}$ It is not surprising that increased postural instability has been documented in patients who experience RD. ${ }^{20}$ Given the high prevalence of RD after successful repositioning and its negative impact on patient recovery, this impairment should be managed properly.

To date, there is no consensus regarding how to effectively manage RD after successful CRP in patients with BPPV. Betahistine is the most widely used medication to ameliorate dizziness due to its effects of increasing labyrinthine microcirculation as well as suppressing the increased neuronal activity in vestibular receptor cells, afferent neurons and vestibular nuclei. ${ }^{11}{ }^{22}$ However, conflicting data exist regarding the efficacy of betahistine for preventing or treating RD. Guneri $e t a t^{23}$ evaluated the efficacy of betahistine in reducing symptoms after Epley's manoeuvre for posterior canal BPPV; they found that $48 \mathrm{mg}$ of daily betahistine was more effective than Epley's manoeuvre alone or combined with a placebo in improving symptoms as assessed by four different vertigo symptoms scales. In contrast, Acar $e t a l^{24}$ carried out a randomised controlled clinical trial to investigate the efficacy of betahistine (compared with a no medication group) for the treatment of RD after successful CRP. After 3 and 5 days of treatment, the mean Dizziness Handicap Inventory (DHI) scores of the groups receiving medication did not differ from the mean DHI score of the control group, indicating that betahistine did not produce greater alleviation of RD than did no treatment. However, both studies have unclear randomisation sequence generation and allocation concealment and only included subjective outcomes. These results suggest that clinical trials with more rigorous methodology and that include comprehensive outcome assessments are warranted.

Recently, non-pharmacological interventions such as vestibular rehabilitation (VR) have been recommended as the first line treatment for chronic vertigo. ${ }^{22} \mathrm{VR}$, which has been available since the 1940s, is an exercise-based treatment consisting of varied eye, head and body movements designed to stimulate the vestibular system and optimise vestibular compensation. ${ }^{25}$ The current VR approach typically includes a combination of three different exercise components to address the impairments or functional limitations identified during evaluation: (1) gaze stability exercises; (2) habituation exercises, including optokinetic exercises and (3) balance and gait training in different conditions. ${ }^{26}$ The aim of VR is to improve the visual-vestibular interaction and increase balance ability. It is also proven to improve daily function and reduce the symptoms of dizziness. ${ }^{26}$ The mechanism underlying its efficacy is thought to rely on the following three aspects: (1) compensation/habituation, a central process referring to the concept that repeated exposure to a provocative stimulus will result in a reduction in the symptomatic response to that treatment ${ }^{27}$; (2) adaptation, which is the recovery of dynamic vestibulo-ocular responses due to the ability of the vestibular system to make long-term changes in the neuronal response to input and (3) substitution, which is the use of other strategies to replace the lost function. ${ }^{28}$

The updated evidence-based clinical guideline of the American Academy of Otolaryngology-Head and Neck Surgery Foundation (AAO-HNSF) indicates that the clinician should offer VR as treatment for BPPV, stating that although movement/habituation based VR should not be a first-line treatment modality for BPPV, it is indicated for patients who experience persistent disability following CRP. ${ }^{29}$ VR is particularly indicated in subjects with additional impairments, such as non-specific dizziness and heightened fall risk. ${ }^{7}$ A Cochrane review ${ }^{30}$ was conducted 
to assess the efficacy of VR in patients with symptomatic unilateral peripheral vestibular dysfunction; this included eight studies investigating VR in BPPV specifically. This review supports the contention that the primary intervention for BPPV should be CRP that directly treat the condition, but movement/habituation-based VR will further aid and benefit long-term functional recovery. A more recent review by Bressi $e t a \vec{l}^{31}$ proposed that a hierarchy of interventions should be offered to patients with BPPV, starting with CRP and followed by post-treatment exercises. The findings also indicated that CRP and VR seem to have a synergic effect in patients with BPPV. ${ }^{31}$

While previous studies have shed light on the benefits of VR in BPPV patients, to date, no randomised clinical trial has been conducted to specifically investigate VR, with or without betahistine, in treating RD following CRP in patients with BPPV. This study has been designed with the broad aim of verifying the efficacy of VR as a firstline treatment option for RD. It conforms to the current recommendations for best clinical practice with respect to the implementation of VR for persons with impairments and functional limitations related to vestibular deficit. ${ }^{26}$ We hypothesise that participants in the VR group will exhibit fewer activity and participation restrictions and less balance dysfunction than participants in the betahistine group. Moreover, we expect that participants in the VR group will experience shorter durations of RD, reduced dizziness-related handicaps and enhanced recovery of otolith dysfunction. We also hypothesise that the effects of VR alone will be non-inferior to a combination of VR and betahistine in terms of the outcome measurements mentioned above.

\section{METHODS AND ANALYSIS}

\section{Study design, setting and participants}

This study was designed as a randomised controlled prospective single-blinded study. Outcome assessment will be conducted by two researchers who are blinded to group allocations.

The study setting is the largest ear, nose and throat (ENT)-specialised hospital in China. The hospital serves a population of $\sim 23$ million persons in Shanghai. It also serves as a referral centre accepting patients from all over the country.

The eligibility criteria are as follows: (1) diagnosis of unilateral BPPV (unilateral posterior semicircular canal BPPV or horizontal semicircular canal BPPV) according to the clinical practice guideline by AAO-HNSF in 2017, ${ }^{29}$ (2) age 18-80 years and (3) residual symptoms after successful repositioning manoeuvres. Participants will be excluded if they (1) are confirmed to have anterior semicircular canal BPPV or multi-canal BPPV; (2) are confirmed to have recurrent BPPV; (3) are diagnosed with coexisting vestibular disorders, including Meniere disease, vestibular neuritis, labyrinthitis and peripheral vestibular loss; (4) are diagnosed with severe cervical spine disease; (5) are diagnosed with severe cardiovascular disease; (6) are diagnosed with known cerebral vascular disease such as carotid stenosis; (7) are pregnant/lactating or planning to become pregnant during the study period or (8) have taken vestibulosuppressants, antihistamines and/or ototoxic medications in the previous 3 months.

\section{Procedure}

Patients who are suspected of having BPPV and visit an ENT specialist are potentially eligible for this study. They will undergo a Dix-Hallpike test, a roll test, and an anterior canal provoking test to confirm whether they are experiencing solely unilateral posterior canal or horizontal canal BPPV. After diagnosis by an ENT specialist, posterior canal BPPV will be treated using Epley's manoeuvre or Semont's liberatory manoeuvre, while horizontal canal BPPV will be treated with barbecue rotation manoeuvres. The same physiotherapist will apply both the testing procedure and the CRP manoeuvre. The CRP is repeated up to three times over the course of 1 day. After a successful repositioning manoeuvre, patients will be asked to complete a DHI test. Patients who fail to respond when up to three CRPs have been performed will not be included in this study.

On the second day following successful CRP, a lead investigator will contact the patient by either telephone or Webchat. Patients will be asked whether they are experiencing residual symptoms. If yes, they will be initially screened via telephone interview, and those who meet the inclusion criteria will be invited to participate in the study. A face-to-face interview will be arranged for patients who express an interest in the trial, written informed consent will be obtained from candidates who agree to participate. After obtaining written consent, the principal investigator (Li HW) will fully review the participant's medical history and perform a physical examination and videonystagmography (VNG) including a caloric test to exclude any central nervous system pathologies or other vestibular diseases. The diagnostic test for BPPV will be repeated to confirm that the exclusionary characters are negative. Pure-tone audiometry and tympanometry will be checked. Gait and balance will also be assessed (Romberg, sharpened Romberg). Whenever necessary, an imaging exam such as a CT scan or MRI will be used to exclude cerebellar disorders. The purpose of the above-mentioned examination is to confirm the diagnosis and validate the homogeneity of all participants who take part in the study. After eligibility screenings, participants will be randomly allocated to one of three groups: Group A patients will perform VR exercises; Group B patients will be prescribed betahistine and Group $\mathrm{C}$ patients will be offered both VR and betahistine. Outcome measurements will be noted at baseline and at 2, 4 and 8 weeks post-randomisation. Outcome measurements will be executed by two independent researchers; one will be responsible for apparatus-based measures and another for subjective data collecting. Both researchers will be unaware of the treatment assignment until the end of the study. A study flowchart is provided in figure 1 . 


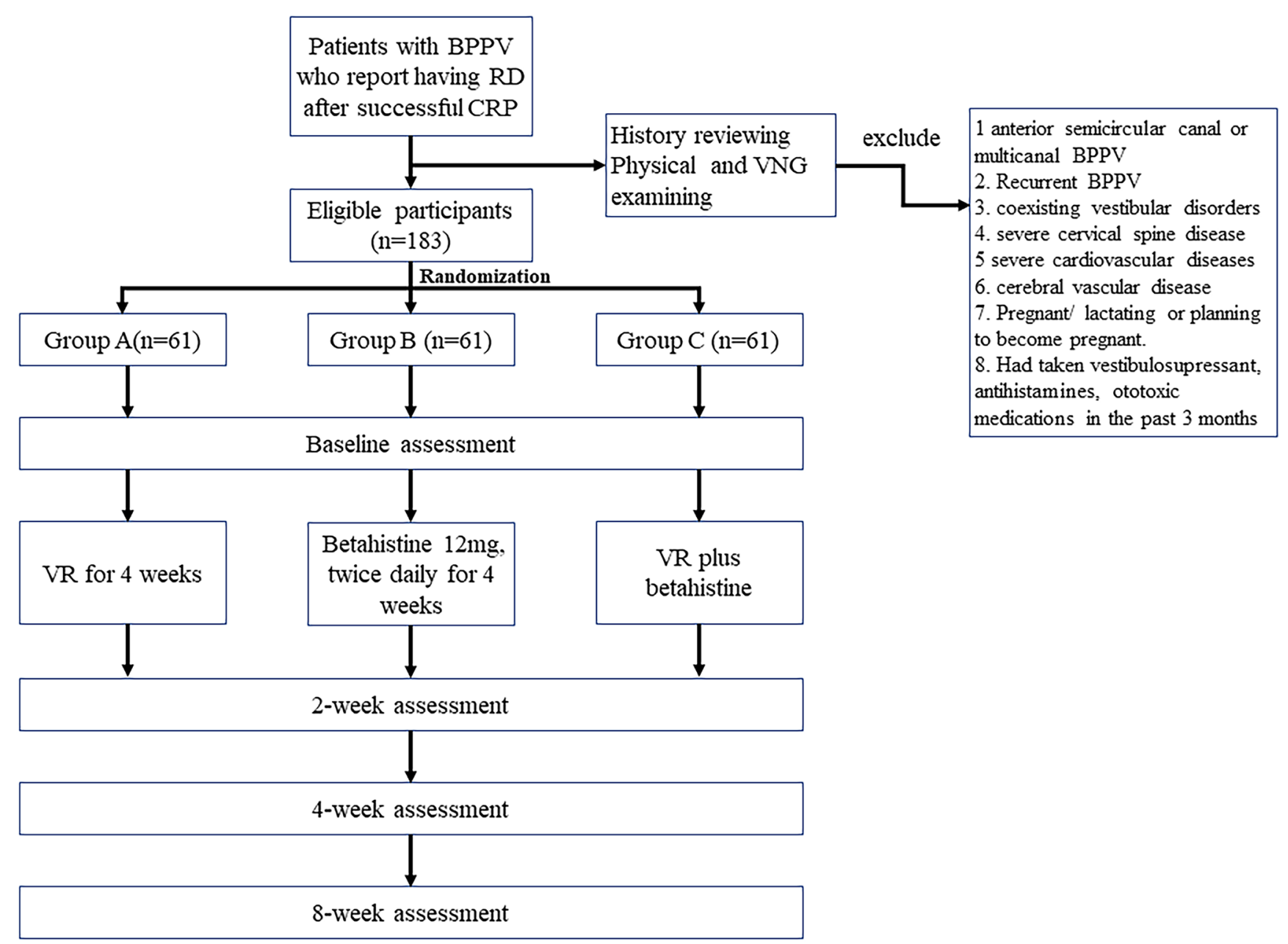

Figure 1 Study flowchart.

\section{Randomisation and blinding}

Participants will be randomly assigned to three groups of 61 persons each. The randomisation process will be carried out by an internet-based randomisation tool provided by the China clinical trial registration centre. The website is accessible to the public via http://www. medresman.org/login.aspx. The randomisation sequence will be generated automatically when the administrator logs into the website and requests the allocation number (once an eligible patient is recruited). Participants will be randomly allocated at a ratio of 1:1:1 and stratified by sex and age ( $\leq 60$ years, $>60$ years). As the randomisation is on-line and automatic, neither the researchers nor the patients can manipulate which treatment the patients will receive; this ensures that group allocations are randomly assigned.

Since VR is an operational intervention, it is not possible for either the patients or the physiotherapists and physicians to be blinded to allocation. However, two independent researchers will contact the patients to collect both subjective and apparatus-based data. The statistician of our trial team will remain blinded until the statistical analyses are complete.

\section{Interventions}

Participants in Group A (VR group) will perform VR exercises twice a day, 7 days per week, over a period of 4 weeks. The exercise protocol was developed based on clinical experience and adapted from previous studies ${ }^{3233}$ in which vestibular stimulation exercise was demonstrated to improve balance ability and functional performance among BPPV patients who had undergone CRP. A dedicated physiotherapist (Wu PX) drafted the initial protocol. The content was reviewed by three experts with experience in caring for patients with chronic dizziness and was then piloted with two female and two male patients who have experienced RD post-BPPV. Participants reported no difficulties in practicing these exercises. The protocol primarily consists of three categories: oculomotor exercises, head movement exercises, and gait and balance training (table 1 ).

The difficulties of the tasks in each session are set according to each participant's balance performance (as measured by computerised dynamic posturography $(\mathrm{CDP}))$ or tolerance to vestibular stimulation. We intend to make the tasks progressively challenging. The approaches used to increase the level of difficulty will be 
Table 1 Overview of the vestibular rehabilitation programme

\begin{tabular}{|c|c|c|}
\hline Components & Movements & Rationale \\
\hline $\begin{array}{l}\text { Oculomotor } \\
\text { exercises }\end{array}$ & $\begin{array}{l}\text { VOR } \times 1 \\
\text { VOR } \times 2 \\
\text { Smooth pursuits } \\
\text { Saccades }\end{array}$ & $\begin{array}{l}\text { Improve gaze stability and VOR gain } \\
\text { adaptation }\end{array}$ \\
\hline $\begin{array}{l}\text { Head } \\
\text { movement } \\
\text { exercises }\end{array}$ & $\begin{array}{l}\text { Shaking the head right and left, nodding the head up and down, } \\
\text { and circumducting the head clockwise and counter clockwise while } \\
\text { maintaining focus on a visual target }\end{array}$ & $\begin{array}{l}\text { Habituate vestibular responses and } \\
\text { relieve symptoms }\end{array}$ \\
\hline
\end{tabular}

VOR, vestibulo-ocular reflex.

as follows: (1) increasing the velocity of exercise performance; (2) changing target distance (near to far) while performing $\mathrm{VOR} \times 1$; (3) placing the target in a distracting visual pattern; (4) performing the exercises with eyes open, then with eyes closed; (5) altering the surface from solid to foam and (6) performing dual tasks such as doing math or talking on a cell phone while walking.

The VR protocol involves one office-based session per week conducted by a skilled physiotherapist (Wu PX) at the clinic and home-based exercises for the remaining 6 days of the week. Compliance will be assessed during home exercise; an exercise diary outlining the time and duration of each exercise is required to be filled out every week. A video and booklet have been developed illustrating the programme and will be used as guides when participants carry out exercise at home. Figures 2 and 3 present the screenshots of the training video; written consent from the person in the video has been obtained. During the first clinical visit, the physiotherapist will provide adequate supervised VR for the patients

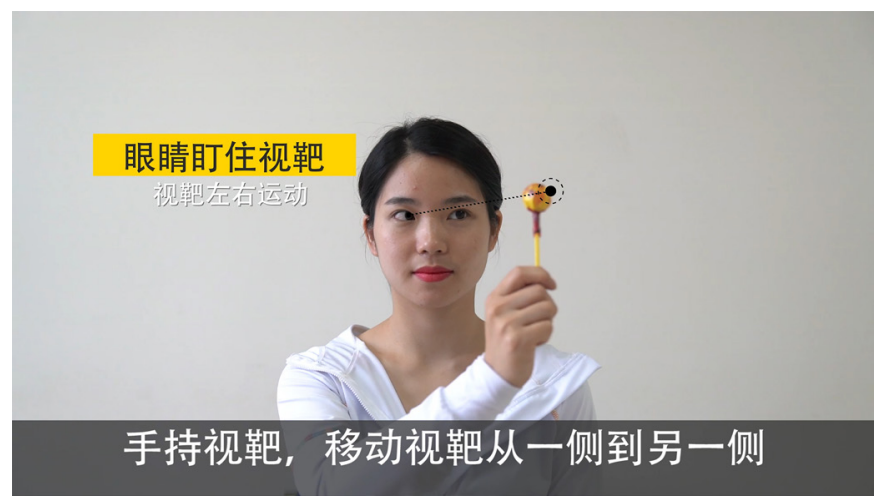

Figure 2 Screenshot of head movement exercises in the training video. to help them understand the goals of the programme and methods to manage their own progress independently. Patients will be instructed not to start any new physical activity during the study period.

Participants in Group B (betahistine group) will be prescribed betahistine $12 \mathrm{mg}$ (manufactured by Wei Cai China Pharmaceutical Co.) (two tablets taken two times per day for 4 weeks). Participants will be asked not to take any other types of antivertiginous medicine during the course of the study; in such a case, the patient should inform his/her ENT specialist. The pharmacy department of the study hospital will be responsible for dispensing betahistine to each participant. There are 30 tablets in one prescription package. Four packages will be prescribed at the commencement of treatment. During the first two follow-up visits ( 2 and 4 weeks post-randomisation), participants will be asked to bring their drug packages and the specialist will count the remaining tablets; if the participant fully adheres to the protocol, eight tablets should be left at the fourth week follow-up visit.

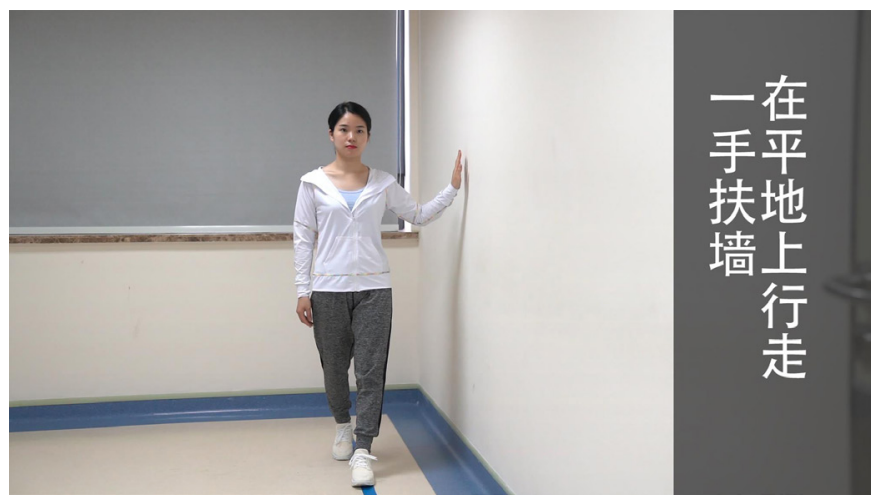

Figure 3 Screenshot of gait exercise in the training video. 
Participants allocated to Group C (VR plus betahistine group) will receive a combination of the VR and betahistine protocols as described above.

\section{Adverse events}

Adverse events are rare while performing VR. However, patients will be instructed to report any complaints or symptoms occurring during or after the exercises. The following symptoms are considered as signs to stop or modify the VR protocol: vomiting, nausea or muscle soreness; a sharp or prolonged pain sensation in the neck, arms or legs; a sensation of ear fullness, hearing loss or tinnitus; double vision or fainting.

The tolerability for betahistine is reported to be good. ${ }^{34}$ Major possible side effects include gastrointestinal system disturbances and headache. ${ }^{35}$ In this study, participants will be encouraged to report any adverse events after betahistine intake. The investigator will routinely contact the participant once per week and ask if any unexpected symptoms have appeared. Any adverse events likely caused by betahistine during the trial will be recorded and reported to the Adverse Drug Reaction administration of the Eye and ENT Hospital of Fudan University within 48 hours.

\section{Withdrawal/retention of participants}

Participation in this study is voluntary and participants have the right to withdraw at any time. However, we will use the approaches recommended by Dziura et $a \hat{l}^{36}$ to improve adherence to the intervention protocols and minimise attrition rates. These include data collection that does not require clinical appointments, a reimbursement mechanism for extra auxiliary examination fees to encourage study completion, and the provision of weekly phone-call contact during the trial. Any concerns, such as unexpected symptoms, as well as logistic issues such as travelling to the clinic or parking issues will be evaluated during each telephone consultation. In rare cases, participants may withdraw owing to unforeseen circumstances. Every reason for withdrawal will be recorded.

\section{Outcome measures}

Evaluating therapeutic success in patients experiencing vestibular disorder is often difficult due to the complexity of this condition and the lack of specific apparatus-based parameters. Various measurements have been utilised; however, no consensus has been reached as to which aspects should be addressed. An international group of investigators and healthcare providers developed a core set based on the International Classification of Functioning, Disability and Health (ICF) framework that illustrates key aspects of functioning that should be measured when assessing patients with vertigo, dizziness, and imbalance. ${ }^{37}$ Of these, two main domains are included: (1) body function and structure and (2) activity and participation. A recently published evidence-based clinical practice guideline has made recommendations for specific rehabilitation outcome assessment by using recommended measures from across the ICF domains. ${ }^{26}$ In the current study, ICF-based activity and participation aspect and apparatus-based measures will be included to provide a full overview of the efficacy of VR on managing $\mathrm{RD}$ after successful repositioning in BPPV patients.

\section{Primary outcomes}

The primary outcomes will focus on patients' daily functional aspects and balance ability, which include:

1. The participant's activity and participation as quantified by the Vestibular Activities and Participation (VAP) scale. The VAP is a 34-item self-reporting questionnaire based on the ICF framework to evaluate the effect of dizziness and/or balance problems on the ability to perform activity and participation tasks. ${ }^{38}$ The difficulty of each task (without assistance from other persons) is rated. Each item of the VAP is scored on a five-point scale indicating the level of difficulty; 0 refers to no difficulty and 4 indicates inability to complete the task. A total score is obtained by calculating the average item scores after excluding the 'not applicable' responses. Previous studies have established the cross-cultural validity of the VAP. ${ }^{39}$ We recently completed the translation and validation of a Chinese version of the VAP.

2. Balance function, as measured by CDP (Equitest, NeuroCom International, Inc.). The Sensory Organisation Test (SOT) of CDP evaluates the ability of subjects to use vision (vestibular and somatosensory) to maintain their balance. ${ }^{40}$ Six increasingly challenging conditions (from SOT1 to SOT6) that disrupt portions of balance sensory input or visual surroundings are provided to evaluate balance. An Equilibrium Score (ES) for each condition is calculated by comparing the angular difference between the subject's calculated maximum and minimum sagittal plane body sway to a theoretical maximum displacement $\left(12.5^{\circ}\right)$. In terms of overall performance, a Composite Score (CS) is given as an estimate of postural stability: a CS score near $100 \%$ indicates little sway, while scores approaching $0 \%$ confirm instability or a fall. The ES for each test condition from SOT1 to SOT6 and the CS will be used for analysis in this study. The values are considered abnormal when the score is lower than the age-specific norm data.

\section{Secondary outcomes}

The secondary outcomes will include dizziness-related handicap, recovery of otolith dysfunction and duration of symptoms.

1. Dizziness-related handicap as measured by the DHI. The DHI has been widely used as a reliable tool for evaluating the self-perceived handicap effects imposed by vestibular system disease and has been translated to Chinese and standardised by Ding et al. ${ }^{41}$ The DHI consists of 25 self-assessment items that can be broken down into three subscales: physical, emotional and functional. Total scores of the DHI range from 0 to 100 with increasing scores indicating greater perception of 
handicap due to dizziness. It has been widely used as a reliable and valid tool for assessing the harmful effects of dizziness on quality of life for patients including those with BPPV. ${ }^{42}$

2. Recovery of otolith function as analysed by Vestibular Evoked Myogenic Potentials (VEMPs). VEMPs are now established as a clinical test of otolith function. The cervical VEMP (cVEMP) is used to assess inferior nerve otolith function (testing mainly saccular function), while the ocular VEMP (oVEMP) is believed to examine superior nerve otolith function (testing mainly utricular function). Previous studies ${ }^{1643}$ identified that, in patients with BPPV who underwent successful CRP, the occurrence of residual symptoms was correlated with oVEMP abnormalities; this suggests that utricular dysfunction is responsible for RD presence. In contrast, another study found that BPPV patients with saccular dysfunction experienced more significant effects than did those with utricular dysfunction. ${ }^{42}$ Therefore, in this study, both cVEMP and oVEMP will be recorded using the Bio-Logic Navigator Pro V.9.0 (Natus Medical, San Carlos, California, USA) system. The procedure will be performed in a sound-proof room. The methodology is reported in detail elsewhere. ${ }^{44}$ Otolith dysfunction in our patients will be defined as a lack of unilateral responses in cVEMPs or oVEMPs.

3. Duration of symptoms as reported by patients. During the trial period, a researcher will contact the participants once per week to ask how many days they experienced symptoms in the previous week. By the end of the study, the number of days per week with reported symptoms will be summed to represent the duration of symptoms.

\section{Time-points of outcome measurements}

Outcome measurements will be performed at baseline and at three follow-up visits. Baseline assessments will be performed on the second day following successful CRP. Demographic and clinical data collected will include: age, sex, education, employment, marital status, coexisting systemic diseases, date of onset, duration of symptoms from onset to treatment, affected ear and canal and the number of CRPs performed. Participants are required to return to the clinic at 2, 4 and 8 weeks post-allocation. They will fill out the same questionnaires (VAP and DHI) at each follow-up, while the CDP and VEMPs will only be repeated when the previous exam indicates abnormality. The schedule of enrolment, interventions, and outcome assessments are presented in table 2 .

\section{Sample size calculation}

The study sample size was based on the VAP measure (one of the primary outcomes), which is a useful clinical tool for assessing a patient's limitations in activities and participation due to vertigo, dizziness and unsteadiness. The validation of VAP in patients who complained of dizziness or imbalance showed the minimum detectable change (MDC) for the VAP was 0.58 , with an SE of $0.21 .^{39}$ In this study, a VAP difference of 0.6 will be considered clinically meaningful. Thus, to detect an MDC of 0.6 for the VAP with $80 \%$ power (alpha level of 0.05 , two-tailed test), 55 subjects per group will be required. Although there are three groups in this study, controlling for the type I error rate is not needed because our hypotheses are: (1) VR will be superior to betahistine (Group A vs Group B) and (2) VR will be non-inferior to VR plus betahistine (Group A vs Group C); therefore, only two comparisons will be executed. Allowing for a $20 \%$ loss during the follow-up period, a total sample of 183 (61 per group) will be required for the current study.

\section{Data analysis}

Statistical analyses will be completed using IBM SPSS Statistics for Windows (SPSS V.22.0) and the level of significance will be set at $\mathrm{p}<0.05$. First, a descriptive analysis will be conducted to determine outliers and the distributions of the data. The three groups will then be analysed with respect to baseline values. Analysis of the outcome measures will be based on the conservative intention-totreat (ITT) approach; moreover, as a secondary analysis, a per protocol analysis will also be performed. Drop-out/ attrition is anticipated. We will examine the structure and pattern of missing data and, if appropriate, multiple imputation methods will be used in both the ITT and the per-protocol analyses.

The main analysis of change in the primary outcomes (VAP score and SOT composite) will use mixed-model with repeated measures (MMRM) analyses of variance (ANOVAs), with group and time as fixed effects and subject as a random effect. Mathematical transformation or categorisation of raw scores will be undertaken to meet the distributional assumptions that are required in MMRM models. Models will adjust for age, sex and other baseline potential confounders. Secondary outcomes include both continuous data (DHI score, duration of symptoms) and dichotomous data (otolith function, defined as normal or abnormal). Analysis of secondary outcomes will be conducted using MMRM ANOVAs for continuous outcomes or logistic regression for dichotomous data, again controlling for baseline potential confounders. Statistical analysis will be carried out by a statistician from Fudan University Medical School, who will be blinded to group allocations and the study hypotheses. To achieve blinding, all participants will be anonymised and study groups will be coded as A, B, C.

\section{Study status and recruitment}

This is protocol V.3.0, updated 20 February 2019. The planned date of first enrolment is 1 April 2019. The estimated time required for recruitment is 15 months. The total duration of this study is expected to be 23 months, including statistical analysis and drafting of the study results. 
Table 2 Schedule of enrolment, interventions and outcome assessments

\begin{tabular}{|c|c|c|c|c|c|}
\hline & \multicolumn{5}{|l|}{ Study period } \\
\hline & $\begin{array}{l}\text { Screening } \\
\text { and eligibility/ } \\
\text { allocation }\end{array}$ & Post-allocation & & & Close-out \\
\hline Time-point & T0 & T1 & T2 & T3 & \\
\hline$(\mathrm{D}=$ day, $\mathrm{W}=$ week $)$ & D2 & W2 & W4 & W8 & \\
\hline Eligibility screening & $\checkmark$ & & & & \\
\hline Medical history review & $\checkmark$ & & & & \\
\hline Physical and VNG examination & $\checkmark$ & & & & \\
\hline Informed consent & $\checkmark$ & & & & \\
\hline Demographic and clinical characteristics & $\checkmark$ & & & & \\
\hline Allocation & $\checkmark$ & & & & \\
\hline \multicolumn{6}{|l|}{ Interventions } \\
\hline VR (Group A) & $\longleftarrow$ & - & $\longrightarrow$ & & \\
\hline Betahistine (Group B) & $\leftarrow$ & - & $\longrightarrow$ & & \\
\hline VR plus betahistine (Group C) & $\longleftarrow$ & - & $\longrightarrow$ & & \\
\hline \multicolumn{6}{|l|}{ Assessments of outcome variables } \\
\hline VAP & $\checkmark$ & $\checkmark$ & $\checkmark$ & $\checkmark$ & \\
\hline CDP & $\checkmark$ & & $\checkmark /{ }^{*}$ & $\checkmark /{ }^{*}$ & \\
\hline $\mathrm{DHI}$ & $\checkmark$ & $\checkmark$ & $\checkmark$ & $\checkmark$ & \\
\hline oVEMP & $\checkmark$ & & $\checkmark /{ }^{*}$ & $\checkmark /{ }^{*}$ & \\
\hline cVEMP & $\checkmark$ & & $\sqrt{ } /{ }^{*}$ & $S /{ }^{*}$ & \\
\hline Symptom duration & & $\checkmark$ & $\checkmark$ & $\checkmark$ & \\
\hline
\end{tabular}

${ }^{*}$ Test is only repeated when the previous exam indicates abnormality.

CDP, computerised dynamic posturography; cVEMP, cervical vestibular-evoked myogenic potential; DHI, Dizziness Handicap Inventory; oVEMP, ocular vestibular-evoked myogenic potential; VAP, Vestibular Activities and Participation; VNG, videonystagmography; VR, vestibular rehabilitation.

\section{Data management}

The Data Safety and Monitoring Board (DSMD) has been organised. The DSMD will monitor the day-to-day running of the trial, review the accumulation data, check the database integrity and be responsible for carrying out interim analyses. The DSMD consists of five persons: a chair, a statistician, a methodologist experienced in research with BPPV, a clinical expert and a patient representative. All members are independent of the study sponsor and report no competing interests. The DSMD will meet approximately once per month from the start of the trial. The trial sponsor will compensate DSMD members for their time and effort, similar to the compensation provided to other trial support personnel.

For data collection, an electronic case report form has been designed to record all of the data at baseline and the three follow-up visits during the trial. Electronic data will be stored on a public platform at http://www. medresman.org, which is sponsored by the China Clinical Trial Registry. The database uses standard techniques to provide security. Access to the database is controlled by user names and encrypted passwords. Individual participant data (IPD) will be made public at 6 months after the completion of the study via the IPD sharing platform.

\section{Patient and public involvement}

Patients and the public were not involved in the design of this study. However, we have consulted with a patient representative about his views on how best to involve patients throughout the proposed project. His views have been incorporated into our revised protocol. Patients and the public will be informed of the study results via peer-reviewed journals or academic conferences.

\section{ETHICS AND DISSEMINATION}

We plan to publish the study findings in peer-reviewed academic journals. We also intend to present this study at local, national, and international conferences where possible. Furthermore, we will draft a summary of the study results to be posted on the website of Eye and ENT hospital that can be accessed by all trial participants as well as relevant interest groups. 
Author affiliations

${ }^{1}$ Department of Nursing, Eye and ENT Hospital of Fudan University, Shanghai, China ${ }^{2}$ Department of Intensive Care Unit, Eye and ENT Hospital of Fudan University,

Shanghai, China

${ }^{3}$ School of Nursing, Fudan University, Shanghai, China

${ }^{4}$ Department of Otorhinolaryngolohy, Eye and ENT Hospital of Fudan University,

Shanghai, China

Contributors All authors have made an intellectual contribution to this protocol. HL was the principal investigator of the trial, with full responsibility for the project. PW, WC and YH conceived the design, developed the protocol and wrote the first draft of this manuscript. $\mathrm{HL}$ and $\mathrm{YH}$ revised and approved the final version of the study protocol and the final manuscript.

Funding This study was supported by Science and Technology Commission of Shanghai Municipality (grant number 184119551900) and the Department of Otorhinolaryngology at Eye and ENT Hospital of Fudan University.

Competing interests None declared.

Patient consent for publication Obtained.

Ethics approval This study was approved by the Institute Review Board of Eye and ENT Hospital of Fudan University (reference number. 2017046).

Provenance and peer review Not commissioned; externally peer reviewed.

Open access This is an open access article distributed in accordance with the Creative Commons Attribution Non Commercial (CC BY-NC 4.0) license, which permits others to distribute, remix, adapt, build upon this work non-commercially, and license their derivative works on different terms, provided the original work is properly cited, appropriate credit is given, any changes made indicated, and the use is non-commercial. See: http://creativecommons.org/licenses/by-nc/4.0/.

\section{REFERENCES}

1. von Brevern M, Radtke A, Lezius F, et al. Epidemiology of benign paroxysmal positional vertigo: a population based study. J Neurol Neurosurg Psychiatry 2007;78:710-5.

2. Kim JS, Zee DS. Clinical practice. Benign paroxysmal positional vertigo. N Engl J Med 2014;370:1138-47.

3. Schuknecht HF, Ruby RR. Cupulolithiasis. Adv Otorhinolaryngol 1973;20:434-43.

4. Epley JM. The canalith repositioning procedure: for treatment of benign paroxysmal positional vertigo. Otolaryngol Head Neck Surg 1992;107:399-404.

5. Brandt T, Steddin S. Current view of the mechanism of benign paroxysmal positioning vertigo: cupulolithiasis or canalolithiasis? J Vestib Res 1993;3:373-82.

6. Inagaki T, Suzuki M, Otsuka K, et al. Model experiments of BPPV using isolated utricle and posterior semicircular canal. Auris Nasus Larynx 2006;33:129-34.

7. Bhattacharyya N, Gubbels SP, Schwartz SR, et al. Clinical practice guideline: benign paroxysmal positional vertigo (Update). Otolaryngol Head Neck Surg 2017;156(3_suppl):S1-S47.

8. Martellucci S, Pagliuca G, de Vincentiis M, et al. Features of Residual Dizziness after Canalith Repositioning Procedures for Benign Paroxysmal Positional Vertigo. Otolaryngol Head Neck Surg 2016;154:693-701.

9. Swain SK BI, Mc S. Role of Ginkgo biloba for controlling residual dizziness after successful treatment of benign paroxysmal positional vertigo: Our experiences at a tertiary care teaching hospital of Eastern India. Int J health Allied Sci 2018;7:196-200.

10. Seok JI, Lee HM, Yoo JH, et al. Residual dizziness after successful repositioning treatment in patients with benign paroxysmal positional vertigo. J Clin Neurol 2008;4:107-16.

11. Giommetti G, Lapenna R, Panichi R, et al. Residual Dizziness after Successful Repositioning Maneuver for Idiopathic Benign Paroxysmal Positional Vertigo: A Review. Audiol Res 2017;7:31-7.

12. Vaduva C, Estéban-Sánchez J, Sanz-Fernández R, et al. Prevalence and management of post-BPPV residual symptoms. Eur Arch Otorhinolaryngol 2018;275:1429-37.

13. Lopez-Escamez JA, Gamiz MJ, Fernandez-Perez A, et al. Long-term outcome and health-related quality of life in benign paroxysmal positional vertigo. Eur Arch Otorhinolaryngol 2005;262:507-11.

14. Korres S, Balatsouras DG, Ferekidis E. Prognosis of patients with benign paroxysmal positional vertigo treated with repositioning manoeuvres. J Laryngol Otol 2006;120:528-33.
15. Faralli M, Lapenna R, Giommetti G, et al. Residual dizziness after the first BPPV episode: role of otolithic function and of a delayed diagnosis. Eur Arch Otorhinolaryngol 2016;273:3157-65.

16. Seo T, Shiraishi K, Kobayashi T, et al. Residual dizziness after successful treatment of idiopathic benign paroxysmal positional vertigo originates from persistent utricular dysfunction. Acta Otolaryngol 2017;137:1149-52.

17. Kim HA, Lee H. Autonomic dysfunction as a possible cause of residual dizziness after successful treatment in benign paroxysmal positional vertigo. Clin Neurophysiol 2014;125:608-14.

18. Staab JP, Eckhardt-Henn A, Horii A, et al. Diagnostic criteria for persistent postural-perceptual dizziness (PPPD): Consensus document of the committee for the Classification of Vestibular Disorders of the Bárány Society. J Vestib Res 2017;27:191-208.

19. Rubenstein LZ, Kenny RA, Eccles M, et al. Preventing falls in older people: new advances and the development of clinical practice guidelines. J R Coll Physicians of Edinb 2003;33:262-72.

20. Abou-Elew MH, Shabana MI, Selim MH, et al. Residual postural instability in benign paroxysmal positional vertigo. Audiol Med 2011;9:8-15.

21. Teggi R, Giordano L, Bondi S, et al. Residual dizziness after successful repositioning maneuvers for idiopathic benign paroxysmal positional vertigo in the elderly. Eur Arch Otorhinolaryngol 2011;268:507-11.

22. van Vugt VA, van der Horst HE, Payne RA, et al. Chronic vertigo: treat with exercise, not drugs. BMJ 2017;358:j3727.

23. Guneri EA, Kustutan O. The effects of betahistine in addition to epley maneuver in posterior canal benign paroxysmal positional vertigo. Otolaryngol Head Neck Surg 2012;146:104-8.

24. Acar B, Karasen RM, Buran Y. Efficacy of medical therapy in the prevention of residual dizziness after successful repositioning maneuvers for Benign Paroxysmal Positional Vertigo (BPPV). B-ENT 2015;11:117-21.

25. Hillier SL, McDonnell M. Vestibular rehabilitation for unilateral peripheral vestibular dysfunction. Cochrane Db Syst Rev 2011;2:CD005397.

26. Hall CD, Herdman SJ, Whitney SL, et al. Vestibular Rehabilitation for Peripheral Vestibular Hypofunction: An Evidence-Based Clinical Practice Guideline: from the American physical therapy association neurology section. J Neurol Phys Ther 2016;40:124-55.

27. Shepard NT, Telian SA, Smith-Wheelock M. Habituation and balance retraining therapy. A retrospective review. Neurol Clin 1990;8:459-75.

28. Herdman SJ, Blatt PJ, Schubert MC. Vestibular rehabilitation of patients with vestibular hypofunction or with benign paroxysmal positional vertigo. Curr Opin Neurol 2000;13:39-43.

29. Bhattacharyya N, Gubbels SP, Schwartz SR, et al. Clinical practice guideline: benign paroxysmal positional vertigo. Otolaryngol Head Neck Surg 2017;156:S1-S47.

30. Hillier SL, McDonnell M. Vestibular rehabilitation for unilateral peripheral vestibular dysfunction. Clin Otolaryngol 2011;36:248-9.

31. Bressi F, Vella P, Casale M, et al. Vestibular rehabilitation in benign paroxysmal positional vertigo: Reality or fiction? Int J Immunopathol Pharmacol 2017;30:113-22.

32. Chang WC, Yang YR, Hsu LC, et al. Balance improvement in patients with benign paroxysmal positional vertigo. Clin Rehabil 2008;22:338-47.

33. Ribeiro KM, Freitas RV, Ferreira LM, et al. Effects of balance Vestibular Rehabilitation Therapy in elderly with Benign Paroxysmal Positional Vertigo: a randomized controlled trial. Disabil Rehabil 2017;39:1198-206

34. Otto V, Fischer B, Schwarz M, et al. Treatment of vertebrobasilar insufficiency-associated vertigo with a fixed combination of cinnarizine and dimenhydrinate. Int Tinnitus J 2008;14:57-67.

35. Murdin L, Hussain K, Schilder AG. Betahistine for symptoms of vertigo. Cochrane Db Syst Rev 2016;6:D10696.

36. Dziura JD, Post LA, Zhao Q, et al. Strategies for dealing with missing data in clinical trials: from design to analysis. Yale $\mathrm{J}$ Biol Med 2013;86:343-58.

37. Grill E, Bronstein A, Furman J, et al. International Classification of Functioning, Disability and Health (ICF) Core Set for patients with vertigo, dizziness and balance disorders. J Vestib Res 2012;22:261-71

38. Alghwiri AA, Whitney SL, Baker CE, et al. The development and validation of the vestibular activities and participation measure. Arch Phys Med Rehabil 2012;93:1822-31.

39. Mueller M, Whitney SL, Alghwiri A, et al. Subscales of the vestibular activities and participation questionnaire could be applied across cultures. J Clin Epidemiol 2015;68:211-9.

40. Nashner LM. Computerized dynamic posturography. In: Jacobson GP SNE, ed. Balance Function Assessment and Management. San Diego: Plural Publishing, 2016. 
41. Ding L, Liu C, Wang JX. An Evaluaiton of the Dizziness Handicap Inventory (Chinese version). Chinese Journal of Otology 2013;11:228-30. In Chinese.

42. Hoseinabadi R, Pourbakht A, Yazdani N, et al. The effects of abnormality of cVEMP and oVEMP on rehabilitation outcomes in patients with idiopathic benign paroxysmal positional vertigo. Eur Arch Otorhinolaryngol 2016;273:643-8.
43. Tian YS, Zhang Y, Ma R, et al. [Features of vestibular evoked myogenic potential in patients with residual dizziness after canalith repositioning procedures for benign paroxysmal positional vertigo]. Lin Chung Er Bi Yan Hou Tou Jing Wai Ke Za Zhi 2018;32:845-9. In Chinese.

44. Zhou YJ, Wu YZ, Cong N, et al. Contrasting results of tests of peripheral vestibular function in patients with bilateral large vestibular aqueduct syndrome. Clin Neurophysiol 2017;128:1513-8. 